\title{
Atypical Neurologic Complications Post Autologous Stem Cell Transplantation
}

Moreb J S ${ }^{1 *}$, Elliott $\mathrm{K}^{1}$ and Verenes $\mathbf{M}^{2}$

${ }^{1}$ Hematologic Malignancies, Transplantation and Cell

Therapy, Novant Health Cancer Institute, Winston-

Salem, North Carolina, USA

${ }^{2}$ Department of Neurology, Neuromuscular Medicine,

Novant Health, Fosyth Medical Center, Winston-Salem,

North Carolina, USA

*Corresponding author: J an S Moreb,

Transplantation and Cell Therapy, Novant Health Cancer Institute, Winston-Salem, North Carolina, USA

Received: March 29, 2021; Accepted: April 16, 2021;

Published: April 23, 2021

\begin{abstract}
We describe the case of a patient with high grade, triple hit, Non-Hodgkin's Lymphoma (NHL) who underwent high-dose chemotherapy and Autologous Stem Cell Transplantation (ASCT) as a consolidation. Patient received BEAM conditioning regimen. She engrafted after usual post ASCT course. However, 2 months post ASCT she developed atypical neurologic symptoms and findings leading to general weakness mainly in the lower extremities with multiple falls, mental status changes and high CSF protein with severe sensorimotor neuropathy. She initially failed treatment with IVIg but responded to high dose steroids. More than one year after transplant, she has maintained her neurological improvement, but unable to walk, while her NHL continues to be in remission.
\end{abstract}

\section{Introduction}

Autologous Stem Cell Transplantation (ASCT) is part of the standard of care for some hematologic malignancies, mainly plasma cell dyscrasias and lymphomas. Neurological complications are more frequent after Allogeneic Hematopoietic Stem Cell Transplantation (Allo-HSCT), but they occur after ASCT [1,2]. Neuromuscular complications have been rarely described after ASCT and usually in the form of case reports or a small number of cases reported in retrospective studies that include patients with allo-HSCT. These include axonal neuropathy [3], brachial plexopathy, polyradiculopathy [4], Chronic Inflammatory Demyelinating Polyneuropathy (CIDP) [5,6], Guillain-Barre Syndrome (GBS) [7], and Immune Mediated Neuropathy (IMN) [8]. Here we report a case of CIDP with atypical presentation early after ASCT in a patient with lymphoma.

\section{Case Presentation}

A 65 yo WF who was healthy except for diabetes mellitus type II, presented about with left neck swelling and left lateral rib pain. CT scans revealed neck, supraclavicular and mediastinal lymphadenopathy. Lymph node biopsy revealed high grade CD10 positive Non-Hodgkin's Lymphoma (NHL) with rearrangements of BCL6, BCL2 and Myc genes detected by FISH studies (triple hit NHL). PET CT scan was performed for staging and revealed extensive hypermetabolic lymphadenopathy throughout the neck, chest, abdomen, and pelvis. The spleen was enlarged with small foci of hypermetabolic lesions, in addition to multiple hypermetabolic bone lesions. Patient completed 6 cycles of dose-adjusted EPOCHRituximab regimen [9]. Repeat PET CT scan showed residual mildly hypermetabolic retroperitoneal lymph nodes (Deauville score 2) with mildly enlarged spleen. Bone marrow biopsy was negative for involvement with disease. The patient underwent consolidation with high dose chemotherapy, using BEAM regimen, and Autologous Stem Cell Transplantation (ASCT). Her length of hospitalization for the transplant was 23 days during which she had the usual complications of diarrhea and neutropenic fever. Upon discharge, she started complaining about back pain and leg pain worse at night with weakness. Her appetite had not recovered and lost about $23 \mathrm{lbs}$ in 4 wks after her discharge. We suspected engraftment syndrome with gastric involvement [10] and she declined a trial of treatment with prednisone. Two months after ASCT, her leg weakness was getting worse and had a fall. Repeat PET CT scan was still negative with Deauville score of 2. Physical therapy was started. At 3 months after ASCT, she presented to the Emergency Room (ER) with increased weakness, dizziness, and multiple falls, unable to stand or walk, and was admitted. Her evaluation included MRIs of the entire neuroaxis which were negative and lumbar puncture with CSF showing albuminocytologic disassociation with high protein of $138 \mathrm{mg} / \mathrm{dL}$, WBC 0 and negative cytology and flow cytometry. She was evaluated for autoimmune process and paraneoplastic neurologic syndrome. These included the following blood tests that were all negative: ANA direct, Sjogren's antibodies, anti-neuronal nuclear antibodies types 1-3, purkinje cell cytoplasmic antibodies types 1-3, AChR ganglionic neuronal antibody, AChR binding antibody, C-ANCA, P-ANCA, atypical pANCA, amphiphysin antibody, CRMP-5-IgG, striational antibody, calcium channel Ab P/Q and $\mathrm{N}$ types, and neuronal (V-G) $\mathrm{K}+$ channel antibody. The CSF was tested for Lyme IgG and IgM, as well as anti-neuronal nuclear antibodies types 1-3 and were negative on two occasions. Her quantitative immunoglobulins were low including IgG $377 \mathrm{mg} / \mathrm{dL}$, IgM $50 \mathrm{mg} / \mathrm{dL}$, IgA $58 \mathrm{mg} / \mathrm{dL}$, IgE $4 \mathrm{mg} /$ dL. The clinical picture was similar to that of GBS with progressive symmetrical weakness involving both legs up to the hips. Thus, IVIg empiric therapy was started at $0.4 \mathrm{gr} / \mathrm{kg} /$ day for 5 days. She had mild improvement in her weakness and was discharged to inpatient rehabilitation. However, one week later she presented with confusion, slow intellect and falling asleep on the phone talking with her daughter. Her chemistry panel showed sodium of 120 which was treated with intravenous saline and salt pills. Patient eventually presented to the ER again with difficulty formulating words and left sided facial weakness. She was admitted to the neuro ICU with suspected brain infarct and received tissue plasminogen activator. However, brain MRI with contrast showed no infarct, only stable small meningioma and chronic small vessel ischemic changes. Video EEG showed no seizure activity but was notable for continuous slowing on the left 
temporal side with rare sharp activity. It was felt that her presentation was due to seizure and was started on Keppra. Cardiac assessment including EKG, echocardiogram and troponins were all negative. Repeat lumbar puncture and CSF examination revealed continued albuminocytologic disassociation with high protein of $106 \mathrm{mg} / \mathrm{dL}$, WBC of 1, and negative serology. CNS vasculitis was considered but eventually it was decided to treat with high dose steroids with the working diagnosis of CIDP or atypical GBS. The patient was bedridden at this point and her neurological exam revealed no reflexes in the Upper (UE) or Lower Extremities (LE), strength was $5 / 5$ in UEs, while in LEs the exam showed hip flexion $4 / 5$, hamstrings and quads $3 / 5$, left foot dorsiflexion $0 / 5$, Rt foot dorsiflexion $3 / 5$, and decreased sensation up to knees. Patient received 5 days of intravenous solumedrol 1 gr daily which was extended to 7 days due to improvement in her LEs strength. She was switched to prednisone $60 \mathrm{mg}$ daily with slow taper. At about 2 wks after starting high dose solumedrol, her left foot dorsiflexion improved to $3 / 5$ and her right foot improved to $5 / 5$, while the rest of the exam remained the same. She underwent continuous rehabilitation with physical therapy without regaining her ability to walk. She has not had any more episodes of confusion or CNS symptoms. About 7 wks after steroids treatment initiated, she had EMG and nerve conduction that showed evidence of a severe subacute-to-chronic generalized sensorimotor neuropathy that primarily affected the legs $>$ arms. No demyelinating features were seen at that time.

Currently, at more than one-year post ASCT, there is no evidence of recurrence of her NHL, and continues on slow tapering of prednisone with IVIg every 4 wks, while maintaining the neurological improvement, and continues to be wheelchair bound. Most recent neurological exam showed improved lower extremity strength but with remaining prominent weakness in her tibialis anterior being $1 / 5$ in right $\mathrm{LE}$ and $0 / 5$ in left $\mathrm{LE}$ and no reflexes.

\section{Discussion}

Our case presented few challenges in making the diagnosis and getting the appropriate treatment. We suspected lymphoma relapse and paraneoplastic syndrome, a known complication of lymphoma, but she was in convincing complete remission and corresponding tests were negative. Her symptoms continued to get worse and the progression of her weakness resembled that of GBS, a rare complication of ASCT $[7,11]$. GBS is a sensory-motor demyelinating acute polyneuropathy. The progression of weakness in this patient was rapid but slower than that usually seen in GBS. EMG was not done at the time due to availability and the patient was treated with IVIg with some improvement. Plasmapheresis was deferred.

Immune-Mediated Neuropathies (IMN) following stem cell transplantation, allogeneic more than autologous, is a recognized entity that includes a group of defined neuropathies including GBS, CIDP and others [8]. Significant morbidity and even mortality could be associated with these neuropathies. In a retrospective study from the Mayo Clinic [8], 12 cases of IMNs were identified in patients who underwent SCT, 6 in ASCT, and 6 in allo-SCT. Four patients had GBS, 7 patients had radiculoplexus neuropathy, and one patient had multiple neuropathies. One ASCT patient died of complications from GBS. Only 4/12 patients, 2 were after ASCT, had these complications within 3 months after SCT. GBS cases happened $\geq 3$ after SCT.
Another report on post SCT GBS described 2 cases, one post ASCT and another post allo-SCT [7], both happened beyond 6 months from transplant.

Immune dysregulation occurring after transplantation is likely the main cause contributing to the neuromuscular complications. These immune dysregulations could be obvious when it is related to graft versus host disease post allogeneic transplants. However often, it is not clear, especially after ASCT. Acute neuropathies that occur within 4 months after ASCT or Allo-SCT have been described in 4 patients among 719 transplant patients over 3 years period in a retrospective study [12]. Three patients were after ASCT for either NHL $(n=2)$ or multiple myeloma $(n=1)$. The diagnoses in these patients included brachial plexopathy $(\mathrm{n}=3)$ and multiple severe bilateral thoracic and lumbosacral radiculopathies $(\mathrm{n}=1)$. Two patients had almost near full recovery, while two had residual deficits on long follow up.

Post ASCT CIDP is not very frequent with only two case reports published. One in MM patient 3-4 wks post ASCT [5], and the other in a patient with multiple sclerosis diagnosed with probable CIDP 9 months after ASCT [6]. Both patients have other reasons for neurological symptoms including treatment with thalidomide in the MM patient whose CIDP symptoms resolved spontaneously. On the other hand, the patient with MS did not respond to treatment with high dose steroids, IVIg and plasmapheresis.

In our case, given the clinical time course, lumbar puncture findings, and response to steroids, we believe the most likely diagnosis is CIDP. The lack of demyelinating features on her EMG are likely a reflection of a couple of factors. First, she had such a severe degree of secondary axonal damage in the legs that no responses could be recorded from the nerves, thus precluding an assessment of conduction velocity. Furthermore, the EMG was done about 2 months after her last admission to the hospital and initiating high dose steroids. Therefore, by the time the EMG was done, her nerves had sufficiently remyelinated such that no significant slowing was seen in the nerves that did have a response on the nerve conduction study. The patient responded to high dose steroids and continues to be in remission on a slow tapering regimen of prednisone and monthly IVIg. We recommend to have more awareness of such infrequent and unique complication that can occur early after ASCT. High dose steroids, IVIg and possibly plasmapheresis should all be considered early in the course of the IMN.

\section{References}

1. Denier C, Bourhis JH, Lacroix C, Koscielny S, Bosq J, Sigal R, et al. Spectrum and prognosis of neurologic complications after hematopoietic transplantation. Neurology. 2006; 67: 1990-1997.

2. Guerrero A, Pérez-Simón JA, Gutierrez N, Caballero D, Ortin F, Gomez Sanchez JC, et al. Neurological complications after autologous stem cell transplantation. Eur Neurol. 1999; 41: 48-50.

3. Boiron JM, Ellie E, Vital A, Marit G, Rème $T$, Vital $C$, et al. Periphera neuropathy after autologous blood stem cell transplantation for multiple myeloma. Leukemia. 1994; 8: 322-326.

4. Rabinstein AA, Dispenzieri A, Micallef IN, Inwards DJ, Litzow MR, Wijdicks EF. Acute neuropathies after peripheral blood stem cell and bone marrow transplantation. Muscle Nerve. 2003; 28: 733-736.

5. Peters G, Larner AJ. Chronic inflammatory demyelinating polyneuropathy after autologous peripheral blood stem cell transplantation. J Peripher Nerv Syst. 2005; 10: 384-385. 
6. Simonsen CS, Hansen G, Piehl F, Edland A. Chronic inflammatory demyelinating polyradiculoneuropathy occurring after autologous haematopoietic stem cell transplantation for multiple sclerosis. Mult Scler J Exp Transl Clin. 2016; 2: 2055217316658304

7. Zhang L, Arrington S, Keung YK. Guillain-Barré syndrome afte transplantation. Leuk Lymphoma. 2008; 49: 291-297.

8. Karam C, Mauermann ML, Johnston PB, Lahoria R, Engelstad JK, Dyck PJ Immune-mediated neuropathies following stem cell transplantation. J Neurol Neurosurg Psychiatry. 2014; 85: 638-642.

9. Dunleavy K, Fanale MA, Abramson JS, Noy A, Caimi PF, Pittaluga S, et al. Dose-adjusted EPOCH-R (etoposide, prednisone, vincristine, cyclophosphamide, doxorubicin, and rituximab) in untreated aggressive diffuse large B-cell lymphoma with MYC rearrangement: a prospective, multicentre, single-arm phase 2 study. Lancet Haematol. 2018; 5: e609-e617.
10. Tzung SP, Hackman RC, Hockenbery DM, Bensinger W, Schiffman K, McDonald GB. Lymphocytic gastritis resembling graft-vs-host disease following autologous hematopoietic cell transplantation. Biol Blood Marrow Transplant. 1998; 4: 43-48.

11. Myers SE, Williams SF. Guillain-Barré syndrome after autologous bone marrow transplantation for breast cancer: report of two cases. Bone Marrow Transplant. 1994; 13: 341-344.

12. Rabinstein AA, Dispenzieri A, Micallef IN, Inwards DJ, Litzow MR, Wijdicks EF. Acute neuropathies after peripheral blood stem cell and bone marrow transplantation. Muscle Nerve. 2003; 28: 733-736. 